\title{
A produção intelectual sobre qualidade da informação na Web of Science entre 1989 a 2016: características básicas
}

\author{
Priscila Basto Fagundes \\ Universidade Federal de Santa Catarina, Departamento de Ciência da Informação, Florianópolis, SC, \\ Brasil \\ priscila.bfagundes@gmail.com
}

William Barbosa Vianna

Universidade Federal de Santa Catarina, Centro de Ciências da Educação, Departamento de Ciência da Informação Florianópolis, SC, Brasil wpwilliam@hotmail.com

Douglas Dyllon Jeronimo de Macedo

Universidade Federal de Santa Catarina, Centro de Ciências da Educação, Departamento de Ciência da Informação Florianópolis, SC, Brasil douglas.macedo@gmail.com

DOI: https://doi.org/10.26512/rici.v11.n3.2018.10388

ARTIGOS DE REVISÃO
Recebido/Recibido/Received: 2017-12-22

Aceitado/Aceptado/Accepted: 2018-05-20

Resumo: O objetivo deste artigo é identificar características bibliométricas básicas relacionadas ao termo "qualidade da informação" na base de dados Web of Science (WoS), no período entre 1989 e 2016. Justifica-se pela importância de fundamentação estruturada sobre o assunto que é desenvolvido em várias áreas do conhecimento. A metodologia utilizada foi de busca simples uma pesquisa na base de dados Web of Science, utilizando-se o termo "information quality", onde obteve-se como resultado 1.326 publicações sobre o tema. A partir deste levantamento serão apresentados: a) a quantidade de artigos publicados divididos por ano de publicação, b) uma evidenciação das principais das palavraschave das publicações, c) os 10 artigos mais citados e, d) a identificação das dimensões relacionadas a qualidade da informação dos artigos mais citados.

Palavras-chave: Bibliometria. Qualidade da informação.

The intellectual production on information quality on the Web of Science between 1989 to 2016: basic characteristics

Abstract: The objective of this article is to identify basic bibliometric features related to the term "quality of information" in the Web of Science (WoS) database, between 1989 and 2016. It is justified by the importance of structured reasoning on the subject that is developed in areas of knowledge. The methodology used was a simple search in the Web of Science database, using the term "information quality", where we obtained as result 1,326 publications on the subject. From this survey will be presented: a) the number of articles published divided by year of publication, b) a disclosure of the main 
keywords of publications, c) the 10 articles most cited, and d) the identification of dimensions related to quality of the most frequently cited articles.

Keywords: Bibliometrics. Information quality.

La producción intelectual sobre calidad de la información en la Web of Science entre 1989 a 2016: características básicas

Resumen: El objetivo de este artículo es identificar características bibliométricas básicas relacionadas al término "calidad de la información" en la base de datos Web of Science (WoS), en el período entre 1989 y 2016. Se justifica por la importancia de una fundamentación estructurada sobre el tema que se desarrolla en varias áreas del conocimiento. La metodología utilizada fue de búsqueda simple una investigación en la base de datos Web of Science, utilizando el término "information quality", donde se obtuvo como resultado 1.326 publicaciones sobre el tema. A partir de este levantamiento se presentarán: a) la cantidad de artículos publicados divididos por año de publicación, b) una evidencia de las principales de las palabras clave de las publicaciones, c) los 10 artículos más citados y, d) la identificación de las dimensiones relacionadas con calidad de la información de los artículos más citados.

Palabras clave: Bibliometría. Calidad de la información.

\section{Introdução}

A informação vem sendo percebida cada vez mais como um recurso importante e indispensável para as organizações e os problemas relacionados a falta de qualidade nas informações geradas e disponibilizadas é um grande desafio a ser considerado no processo de gestão da informação.

O conceito de qualidade da informação vem sendo discutido por acadêmicos e profissionais em diferentes maneiras e perspectivas. De acordo com Wang e Strong (1996), o termo qualidade da informação é definido como sendo "aptidão ao uso" e sugere que duas dimensões sejam consideradas: a) o primeiro é que a qualidade da informação depende da tarefa que está sendo executada, uma vez que, um usuário pode considerar a informação apropriada para uma tarefa, mas não suficiente para outra e; b) a segunda, é que a qualidade da informação é subjetiva, dado que um segundo usuário pode considerar a mesma informação apropriada para ambas as tarefas. Sendo assim, ao contrário de outros produtos em que a qualidade pode ser definida de uma forma mais objetiva e exclusiva, a qualidade da informação varia de acordo com a perspectiva do usuário (BIZER; CYGANIAK, 2009).

Para Calazans (2009), uma das motivações em relação à estudos sobre a produção, organização, acesso e uso da informação se deve por ela estar inserida nos mais diversos campos do conhecimento. Da mesma forma que os atributos, é possível encontrar na literatura diversos modelos para serem utilizados no processo de avaliação da qualidade nos mais diferentes contextos, como por exemplo: internet, saúde, sistemas de informação coorporativos, egovernment entre outros. No que tange a área da Ciência da Informação, é importante ressaltar 
o envolvimento do profissional da informação nas discussões sobre a qualidade da informação, uma vez que a mesma é o principal objeto de estudos da área. Outra questão a se considerar remete à diversidade de informações disponíveis, uma vez que são geradas a partir de fontes distintas causando uma sobrecarga de informação para a sociedade, e consequentemente gerando inúmeras oportunidades de atuação para os profissionais que atuam na área da gestão da informação (RIBEIRO, 2014).

Nesse sentido, pergunta-se: quais as características e o comportamento da produção intelectual sobre "qualidade da informação" indexada na Web of Science (WoS)?

O objetivo deste artigo é apresentar um estudo bibliométrico básico sobre as publicações relacionadas ao tema qualidade da informação e para isso foi realizada uma pesquisa na base de dados Web of Science, utilizando-se o termo "information quality", onde obteve-se como resultado 1.326 publicações sobre o tema.

A partir deste levantamento serão apresentados: a) a quantidade de artigos publicados no período entre 1989 e 2016 divididos por ano de publicação, b) uma evidenciação das principais das palavras-chave das publicações, c) os 10 artigos mais citados e, d) a identificação das dimensões relacionadas a qualidade da informação dos artigos mais citados.

Pretende-se com esse estudo, contribuir com novas pesquisas acerca da qualidade da informação, uma vez que irá apresentar uma análise quantitativa das produções científicas na área, bem como uma síntese dos objetivos e das dimensões utilizadas para avaliação da qualidade da informação dos estudos mais referenciados na Web of Science.

\section{Breve problematização sobre qualidade da informação}

Em 1989, o Nordic Concil for Scientific Information and Research Libraries (NORDINFO) promoveu na Dinamarca, um seminário onde ocorreram diversas discussões acerca da qualidade da informação. A publicação decorrente deste encontro é considerada um marco de referência sobre o assunto e foi reconhecida como o esforço mais importante de teorização sobre o tema na época (PAIN et al, 1996).

Um dos pesquisadores participantes do seminário na Dinamarca foi J. Olaisen, e para ele a qualidade da informação se divide em dois grupos filosóficos, são eles: fatores de qualidade de autoridade cognitiva para o usuário, que foca em 'como' a informação seria percebida, e fatores de amigabilidade técnica com o usuário, que dependem 'do que é' oferecido.

J. Olaisen, propõe então, um modelo onde os aspectos usuais da qualidade são agrupados em quatro categorias: qualidade cognitiva, qualidade do desenho da informação, fatores referentes ao produto da informação e fatores relativos à qualidade da transmissão. $\mathrm{A}$ 
'qualidade cognitiva' está relacionada em como a fonte que gerou a informação é valorizada pelo usuário. Nessa categoria, incluem-se os seguintes aspectos: credibilidade, relevância, confiança, validade e significado no tempo. A 'qualidade do desenho da informação', incorpora fatores referentes à forma, flexibilidade e seletividade.

Os fatores referentes 'ao produto da informação', são valor real e abrangência e, finalmente, os fatores relativos à 'qualidade da transmissão' são definidos pelo critério da acessibilidade. $O$ conjunto dos fatores e a relação entre eles configuram o que o autor chama de processo de qualidade da informação (OLAISEN, 1989 apud NEHMY, 1996).

Outro estudo com o propósito de identificar as dimensões da qualidade da informação com base na percepção dos usuários foi realizado por Wang e Strong (1996), onde a partir de uma pesquisa empírica, os autores identificaram um conjunto de 15 dimensões que foram divididas em quatro categorias: A categoria denominada intrínseca, trata da precisão das informações e contempla as dimensões relacionadas a precisão, objetividade, credibilidade e fidelidade, a categoria contextual refere-se a relevância das informações e contém as dimensões: relevância, valor agregado, atualização, completeza e valor apropriado, a categoria representacional, está relacionada a forma de representação das informações e envolve as dimensões: interpretabilidade, facilidade de entendimento, representação concisa e representação consistente e a categoria acessibilidade que diz respeito as formas de acesso às informações.

A partir da pesquisa de Wang e Strong (1996), várias outras proposições relacionadas a dimensões e atributos da qualidade da informação, foram realizadas. No entanto, Batini et al. (2009) argumenta, que não existe um acordo quanto ao conjunto de dimensões que definem a qualidade nem da informação, nem do significado exato de cada dimensão.

Um estudo que corrobora com o pensamento de Batini et al. (2009), foi realizado em 2011. Neste, foi feito um levantamento dos atributos da qualidade da informação, por meio da técnica de análise de conteúdo em artigos de periódicos e atas de eventos científicos publicados entre 1974 e 2009 que identificou a ocorrência de 101 termos distintos referentes aos atributos de qualidade da informação. Destes, foram contabilizados 40 termos com mais de uma ocorrência e 61 termos com apenas uma ocorrência nas referências pesquisadas (AROUCK, 2011). A alta quantidade de atributos identificados neste estudo vem confirmar a falta de consenso no que diz respeito aos critérios utilizados para mensurar a informação.

Segundo Valente e Fujino (2016), os critérios e dimensões da qualidade da informação variam de acordo com as abordagens e vertentes sob as quais os estudos são realizados e de acordo com o contexto onde o conceito de qualidade é aplicado e avaliado. Para as autoras, a qualidade da informação pode ser considerada como uma categoria multidimensional dentro 
deste contexto, uma vez que não se observa na literatura uma conformidade sobre o tema, existindo uma menção recorrente entre os autores interessados neste assunto, que afirmam que as definições propostas sobre qualidade da informação são ainda ambíguas, vagas ou subjetivas.

Por estas breves considerações pode-se verificar a relevância de um aprofundamento daquilo que vem sendo considerado sobre o tema na literatura qualificada. Essa investigação poderá colaborar para estudos futuros sistematizados sobre as características, dimensões, principais ocorrências e desenvolvimento do tema na literatura, dada sua polissemia.

\section{Procedimentos e opções metodológicas}

Para a realização do estudo bibliométrico, foi escolhida a base de dados Web of Science. A escolha se deu, pela mesma ser considerada uma base de referência multidisciplinar que está integrada à base de dados ISI Web of Knowledge, bem como por ser considerada uma importante indexadora de periódicos científicos que oferece ferramentas para análise de citações, referências, índice h, permitindo a realização de análises bibliométricas (PORTAL DE PERIÓDICOS DA CAPES, 2017; LUCAS; FREDD; VIERA, 2015)

A consulta foi realizada em junho de 2017 e utilizou-se o termo "information quality", utilizando o campo de pesquisa Tópico que engloba a consulta ao título, ao resumo e as palavras-chave. Quanto ao período de publicação, foram considerados os estudos publicados entre o período de 1989 a 2016 e quanto ao tipo de documento foram selecionados apenas os artigos indexados pela Web of Science, excluindo os proceedings paper, book review, editorial material e review entre outros de menor ocorrência.

Para a realização da análise dos dados resultantes da pesquisa foram utilizados os recursos de análise disponibilizados pela Web of Science que possibilitou a geração de gráficos e de normalização dos dados em conjunto com a ferramenta Excel da Microsoft, versão 2016.

\section{Dados sobre qualidade da informação na Web of Science}

A pesquisa realizada na Web of Science, que cobre aproximadamente 12.000 periódicos, com o termo "information quality" durante o período de 1989 a 2016, e recuperou 1.326 artigos. Os dados sobre os autores, palavras-chaves, ano de publicação e qual o periódico da publicação, foram extraídos para o Excel para que fosse possível uma melhor análise, onde os resultados gerais apresentaram que os 1.326 que foram produzidos por 3.824 autores ${ }^{1}$ e publicados em 736 periódicos científicos distintos.

\footnotetext{
${ }^{1}$ A partir de uma análise preliminar na lista de autores, foram identificados casos que um mesmo autor pode ter sido referenciado de maneiras diferentes, como por exemplo, Abouassaly, R. e Abouassaly, Robert. Salienta-se que
} 
Gráfico 1. Quantidade de publicações por ano.

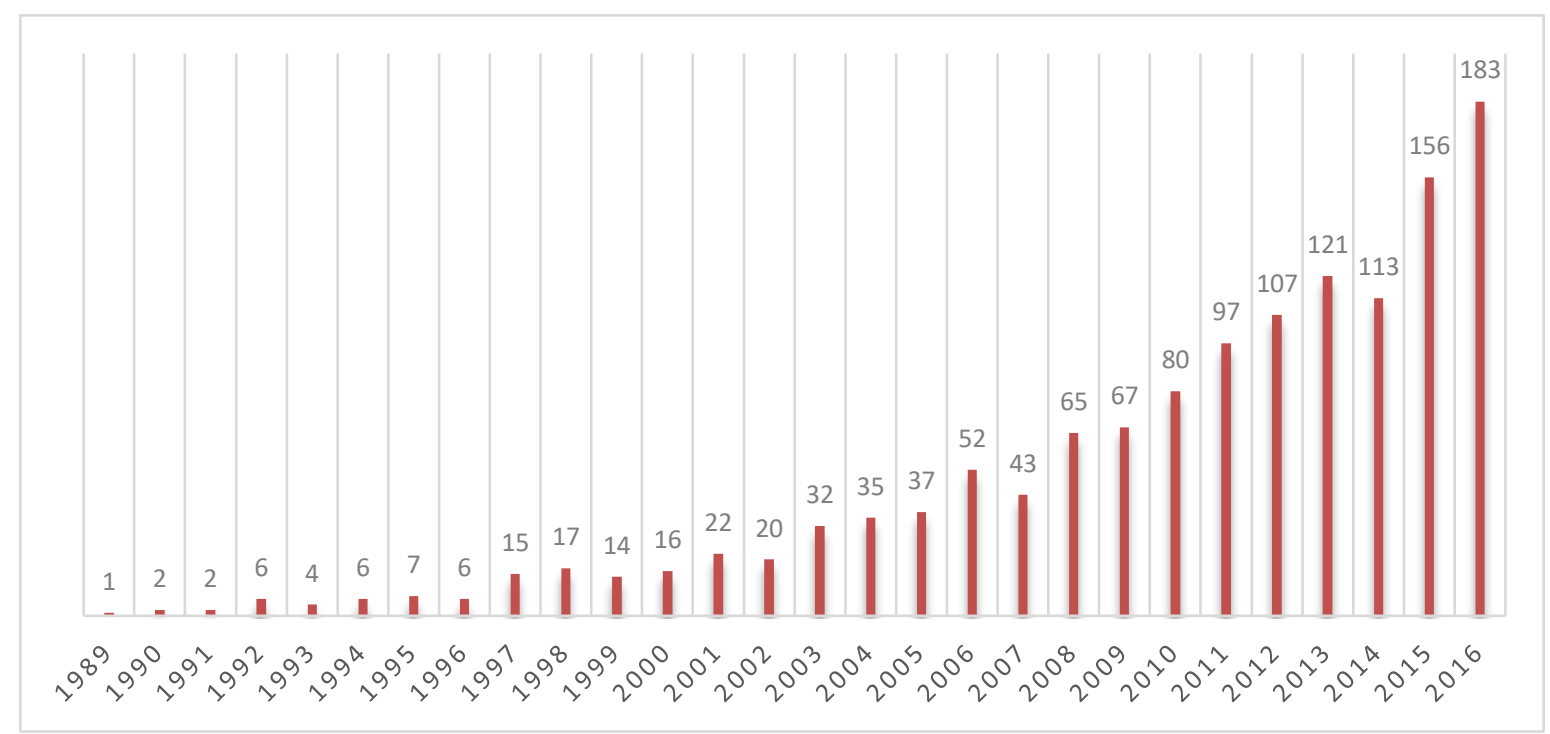

Fonte: dados da pesquisa na WoS

Observando o Gráfico 1, nota-se que o número de publicações encontradas na base de dados Web of Science, obteve um aumento gradativo ao longo dos anos, com exceção dos anos de 1993, 1996, 1999, 2002, 2007 e 2014, onde o número de publicações diminuiu de um ano para o outro, porém em quantidades não significativas.

O aumento de publicações do ano de 2014 para o de 2015, é o mais representativo indo de 113 para 156 respectivamente. Acredita-se que o crescimento de pesquisas na área nos últimos anos mostra a preocupação da comunidade acadêmica em propor soluções que garantam uma maior qualidade das informações disponibilizadas.

Por meio da ferramenta Excel, foi realizada a separação de todas as palavras-chaves contidas nas 1.326 publicações, contabilizando um total de 5.364 termos, após a remoção dos registros duplicados obteve-se 2.536 termos distintos. O processo de contagem das palavras se deu através da cópia da lista das palavras-chaves geradas pela Web of Science para uma planilha do Excel, onde foram retirados todos os espaços em branco contidos entre os caracteres ";", localizados antes e depois das palavras-chave, após, as mesmas foram transformadas em strings apenas contendo caracteres minúsculos e por fim foram eliminadas todas as duplicações.

O Quadro 1 apresenta uma síntese do levantamento realizado em relação a quantidade de palavras-chave, sob o total das 5.364. Serão apresentadas a quantidade de palavras-chave, quantas vezes a palavra apareceu na contagem e uma amostra das palavras

para este estudo, na possibilidade da ocorrência destes casos, a contagem final considerou esses registros como se fossem autores distintos. 
encontradas (quando a quantidade for maior que 5, caso contrário serão apresentadas todas as palavras).

Quadro 1. Síntese dos resultados das palavras-chaves.

\begin{tabular}{|c|c|c|}
\hline $\begin{array}{l}\text { Qtd } \\
\text { palavras }\end{array}$ & $\begin{array}{l}\text { Qtd ocorrência } \\
\text { de cada palavra }\end{array}$ & Palavras-chaves \\
\hline 3037 & 1 & accounting quality; advocacy shanghai; social; high school \\
\hline 281 & 2 & tourism; xml; social networking; culture; ontology \\
\hline 127 & 3 & supply chain; gender; e-business; financial reporting; health services \\
\hline 51 & 4 & web; metadata; e-service quality; cancer; business intelligence \\
\hline 20 & 5 & $\begin{array}{l}\text { management; information quantity; Australia; mobile banking; } \\
\text { reliability }\end{array}$ \\
\hline 15 & 6 & website; online shopping; e-government; assessment; performance \\
\hline 11 & 7 & $\begin{array}{l}\text { world wide web; quality assessment; IS success; e-learning; knowledge } \\
\text { management }\end{array}$ \\
\hline 6 & 8 & $\begin{array}{l}\text { usability; perceived usefulness; information system; information } \\
\text { exchange; information asymmetry }\end{array}$ \\
\hline 2 & 9 & learning; health information \\
\hline 5 & 10 & $\begin{array}{l}\text { website quality; structural equation modeling; quality of information; } \\
\text { patient education; information technology }\end{array}$ \\
\hline 3 & 11 & $\begin{array}{l}\text { technology acceptance model; information systems success; decision } \\
\text { making }\end{array}$ \\
\hline 4 & 13 & $\begin{array}{l}\text { social media; information management; evaluation; customer } \\
\text { satisfaction }\end{array}$ \\
\hline 1 & 14 & information sharing \\
\hline 2 & 17 & supply chain management; e-commerce \\
\hline 3 & 18 & satisfaction; IS success model; information systems \\
\hline 1 & 26 & information \\
\hline 1 & 31 & data quality \\
\hline 1 & 33 & quality \\
\hline 2 & 34 & user satisfaction; trust \\
\hline 1 & 50 & service quality \\
\hline 1 & 61 & system quality \\
\hline 1 & 67 & internet \\
\hline 1 & 274 & information quality \\
\hline
\end{tabular}

Fonte: dados da pesquisa na WoS 
Conforme pôde ser observado, existe um elevado número de palavras-chave que apresentam poucas ocorrências. Foi realizada uma análise nas 3.037 que apareceram apenas uma vez e constatou-se que a maioria delas eram bastante específicas, como por exemplo: "Spanish universities", "South Africa", "Portugal", "Hong Kong", fazendo referência à região onde o estudo foi aplicado.

Outra característica é que as palavras-chaves apresentaram referências aos contextos de aplicação da qualidade da informação, por exemplo: "public health", "Google maps" e "education", o que indica a ampla possibilidade de estudos e pesquisas. Já em relação as palavras-chave que apresentaram mais de 30 ocorrências, entre elas: "quality", "service quality", "system quality", "internet", e "information quality", pode-se dizer que apesar de abrangentes, são significativas na área da qualidade da informação, características que podem ter contribuído para o alto número de ocorrências.

O Quadro 2 apresenta informações sobre as 10 publicações mais citadas na área da qualidade da informação. Serão apresentadas, o título da publicação, o nome dos autores, o ano, o periódico em que o artigo foi publicado e o número de citações da obra. Vale ressaltar que os dados relacionados aos artigos mais citados e suas quantidades de citações, diferentes dos dados apresentados anteriormente, são dinâmicos e poderão sofrer alterações se consultados no futuro, uma vez que os mesmos podem ser referenciados por novos estudos a qualquer momento a partir da data do levantamento realizado.

Quadro 2. Artigos mais citados na área da qualidade da informação de acordo com dados da Web os Science

\begin{tabular}{|c|c|c|c|c|c|}
\hline & Título & Autores & Periódico & Ano & Citações \\
\hline 1 & $\begin{array}{l}\text { Information Systems Success: The Quest } \\
\text { for the Dependent Variable }\end{array}$ & $\begin{array}{l}\text { DeLone, W. H.; } \\
\text { McLean, E R }\end{array}$ & $\begin{array}{l}\text { Information } \\
\text { Systems } \\
\text { Research }\end{array}$ & 1992 & 2250 \\
\hline 2 & $\begin{array}{l}\text { The DeLone and McLean model of } \\
\text { information systems success: a ten-year } \\
\text { update }\end{array}$ & $\begin{array}{l}\text { DeLone, W. H; } \\
\text { McLean, E. R }\end{array}$ & $\begin{array}{l}\text { Journal of } \\
\text { Management } \\
\text { Information } \\
\text { Systems }\end{array}$ & 2003 & 1964 \\
\hline 3 & $\begin{array}{l}\text { A theoretical integration of user } \\
\text { satisfaction and technology acceptance }\end{array}$ & $\begin{array}{l}\text { Wixom, B. H; Todd, } \\
\text { P.A }\end{array}$ & $\begin{array}{l}\text { Information } \\
\text { Systems } \\
\text { Research }\end{array}$ & 2005 & 585 \\
\hline 4 & $\begin{array}{l}\text { The measurement of web-customer } \\
\text { satisfaction: An expectation and } \\
\text { disconfirmation approach }\end{array}$ & $\begin{array}{l}\text { McKinney, V; Yoon, } \\
\text { K; Zahedi, F }\end{array}$ & $\begin{array}{l}\text { Information } \\
\text { Systems } \\
\text { Research }\end{array}$ & 2002 & 557 \\
\hline 5 & $\begin{array}{l}\text { IBM Computer Usability Satisfaction } \\
\text { Questionnaires - Psychometric }\end{array}$ & Lewis, J.R & $\begin{array}{l}\text { International } \\
\text { Journal of }\end{array}$ & 1995 & 455 \\
\hline
\end{tabular}




\begin{tabular}{|c|c|c|c|c|c|}
\hline & Evaluation and Instructions for Use & & $\begin{array}{l}\text { Human- } \\
\text { Computer } \\
\text { Interaction }\end{array}$ & & \\
\hline 6 & $\begin{array}{l}\text { A trust-based consumer decision-making } \\
\text { model in electronic commerce: The role } \\
\text { of trust, perceived risk, and their } \\
\text { antecedents }\end{array}$ & $\begin{array}{l}\text { Kim, D. J.; Ferrin, D } \\
\text { L.; Rao, H. R }\end{array}$ & $\begin{array}{l}\text { Decision } \\
\text { Support } \\
\text { Systems }\end{array}$ & 2008 & 433 \\
\hline 7 & Costs of equity and earnings attributes & $\begin{array}{l}\text { Francis, J; LaFond, R; } \\
\text { Olsson, P. M; } \\
\text { Schipper, K }\end{array}$ & $\begin{array}{l}\text { Accounting } \\
\text { Review }\end{array}$ & 2004 & 421 \\
\hline 8 & $\begin{array}{l}\text { Assessing the validity of IS success } \\
\text { models: An empirical test and theoretical } \\
\text { analysis }\end{array}$ & $\begin{array}{l}\text { Rai, A; Lang, S.S; } \\
\text { Welker, R.B }\end{array}$ & $\begin{array}{l}\text { Information } \\
\text { Systems } \\
\text { Research }\end{array}$ & 2002 & 386 \\
\hline 9 & $\begin{array}{l}\text { The technology acceptance model and } \\
\text { the World Wide Web }\end{array}$ & $\begin{array}{l}\text { Lederer, A.L; Maupin, } \\
\text { D.J; Sena, M.P; } \\
\text { Zhuang, Y.L }\end{array}$ & $\begin{array}{l}\text { Decision } \\
\text { Support } \\
\text { Systems }\end{array}$ & 2000 & 360 \\
\hline $\begin{array}{l}1 \\
0\end{array}$ & $\begin{array}{l}\text { Accounting information, disclosure, and } \\
\text { the cost of capital }\end{array}$ & $\begin{array}{l}\text { Lambert, R.; Leuz, C.; } \\
\text { Verrecchia, R. E }\end{array}$ & $\begin{array}{l}\text { Journal of } \\
\text { Accounting } \\
\text { Research }\end{array}$ & 2007 & 353 \\
\hline
\end{tabular}

Fonte: dados da pesquisa na WoS.

As primeiras duas publicações mais citadas encontradas na Web of Science são de autoria de DeLone e McLean, a primeira foi publicada em 1992 e foca na qualidade dos sistemas de informação como um todo e propõe que estes sejam analisados através de seis categorias: qualidade do sistema, qualidade de informação, uso, satisfação do usuário, impacto individual e impacto organizacional.

No que tange a qualidade da informação e com base em outros estudos realizados anteriormente, DeLone e McLean (1992), sugerem um modelo para medir a qualidade da informação a partir da perspectiva dos usuários que contempla 23 dimensões: importância, relevância, utilidade, representatividade, usabilidade, compreensibilidade, legibilidade, clareza, formato, aparência, conteúdo, exatidão, precisão, concisão, suficiência, completude, confiabilidade, aceitação, atualidade, singularidade, comparabilidade, quantificação e objetividade.

A segunda publicação mais citada, também de DeLone e McLean, apresenta uma discussão sobre as contribuições feitas por outros pesquisadores ao modelo proposto em 1992, e com base na avaliação destas contribuições os autores recomendam uma atualização no modelo proposto anteriormente. Neste, os autores sugerem uma alteração em relação as categorias. 
As categorias relacionadas a qualidade do sistema, qualidade de informação, uso e satisfação do usuário, permaneceram inalteradas, sendo retiradas as categorias: impacto individual e impacto organizacional. No lugar destas foram incluídas, qualidade do serviço e "net benefits" - optou-se aqui por manter o termo em inglês. Não consta no artigo nenhuma referência de atualização nas 23 dimensões da categoria qualidade da informação, citadas anteriormente, porém, é feita uma proposição de aplicação do modelo no contexto $e$ commerce, e na descrição desta aplicação entendeu-se que as dimensões podem ser definidas e utilizadas de acordo com o contexto de aplicação. Para o contexto de e-commerce o modelo sugere a utilização das dimensões: completude, compreensibilidade, personalização, relevância e segurança.

Em Wixon e Todd (2005), a terceira publicação mais citada, é apresentado um modelo de avaliação da qualidade de sistemas de informação que visa contemplar a qualidade da informação bem como a qualidade do sistema, ambas sob a perspectiva do usuário. Os autores sugerem que para medir a qualidade da informação sejam levados em consideração características como, completude, precisão, formato e atualização. Também objetivando a avaliação da qualidade da informação e a qualidade do sistema, porém com o foco em lojas online, o artigo de Mckinney; Yoon e Zahedi (2002), que está em quarto lugar na lista dos mais citados, propõe que o conjunto de fatores de análise da qualidade da informação contemplem, a relevância, a compreensibilidade, a confiabilidade, a adequação, o escopo e a utilidade.

A quinta publicação mais citada pertence a Lewis (1995), e apresenta uma discussão acerca das qualidades psicométricas dos questionários utilizados por quatro métodos para avaliação da satisfação dos usuários em relação a usabilidade dos sistemas de informação. De acordo com Nunnally, (1978 apud Lewis, 1995), a psicometria é usada para medir de maneira apropriada e através de comprovação experimental, um conjunto de comportamentos ao qual se deseja um melhor entendimento.

Os métodos analisados no artigo citado foram: o ASQ - After-Scenario Questionnaire, o PSQ - Printer Scenario Questionnaire, o PSSUQ - Post-Study System Usability Questionnaire e o CSUQ - Computer System Usability Questionnaire. Por se tratar de um estudo com foco na qualidade psicométrica de questionários, as dimensões analisadas estão fora do contexto das dimensões da qualidade da informação e por este motivo não farão parte da análise apresentada neste artigo.

Outro estudo com foco na qualidade da informação em sistemas e-commerce é apresentado em Kim; Ferrin e Rao (2008). O objetivo do sexto artigo mais citado, foi o desenvolvimento de um quadro teórico descrevendo o processo para a tomada de decisão do consumidor ao fazer uma compra em um determinado site. Os resultados mostraram que 
fatores como a reputação da empresa, questões relacionadas a privacidade e a segurança, e a qualidade das informações contidas no site, têm fortes influências na confiabilidade dos consumidores no site de e-commerce. De acordo com as métricas propostas, as dimensões da qualidade da informação consideradas relevantes neste contexto são exatidão, utilidade, atualidade, suficiência, satisfação.

Francis et al. (2004), apresentam em sua publicação, a sétima mais citada, uma análise baseada em modelos teóricos contábeis que estabelecem uma relação entre a qualidade das informações específicas das empresas, como por exemplo seu lucro, dividendos, entre outros e o custo da equidade. Por a qualidade da informação não ser o foco do estudo, as dimensões da qualidade da informação não são contempladas. A publicação de Rai (2002), na oitava colocação entre as mais citadas, ao contrário de todos os outros artigos apresentados, não está disponível através de acesso aberto para leitura integral, por esse motivo, o mesmo não fará parte desta análise.

A nona publicação mais citada é de Lederer et al. (2000) e contempla a validação do modelo TAM - Technology Acceptance Model no contexto da web. O modelo não trata especificamente da qualidade da informação, e sim de um modelo a ser aplicado nos sistemas com o objetivo de prever se o mesmo terá uma boa aceitação por parte dos usuários. 0 resultado possibilita que desenvolvedores e gestores antecipem futuros problemas relacionados a receptividade do sistema. A qualidade da informação é um dos fatores analisados no modelo proposto e os atributos considerados relevantes dizem respeito a relevância, a precisão, a pontualidade, a compreensibilidade e a exatidão da informação.

Outra publicação com o foco nas informações contábeis, pertence a Lambert; Leuz e Verrecchia (2007), e seu objetivo é verificar como a qualidade das informações contábeis disponibilizadas pelas empresas podem influenciar direta e indiretamente no custo do capital. Da mesma forma que a publicação de Francis et al. (2004), a décima publicação mais citada, não indica quais as características das informações devem ser observadas para que os relatórios contábeis disponibilizados sejam considerados de qualidade.

A seguir, o Quadro 3 apresenta uma síntese da análise realizada em cada uma das 10 publicações mais citadas na base de dados Web of Science. Serão apresentados o contexto de aplicação do estudo e as dimensões propostas. Para algumas publicações as informações sobre as dimensões estarão em branco, isto se deve ao fato das mesmas não terem sido identificadas nos respetivos estudos. 
Quadro 3. O contexto de aplicação e as dimensões da qualidade da informação dos artigos mais citados na Web of Science

\begin{tabular}{|c|c|c|c|}
\hline & Autores & $\begin{array}{l}\text { Contexto de } \\
\text { Aplicação }\end{array}$ & Dimensões \\
\hline 1 & $\begin{array}{l}\text { DeLone e } \\
\text { McLean, (1992) }\end{array}$ & $\begin{array}{l}\text { Sistemas de } \\
\text { Informação (SI) }\end{array}$ & $\begin{array}{l}\text { importância, relevância, utilidade, representatividade, } \\
\text { usabilidade, compreensibilidade, legibilidade, clareza, } \\
\text { formato, aparência, conteúdo, exatidão, precisão, concisão, } \\
\text { suficiência, completude, confiabilidade, aceitação, } \\
\text { atualidade, singularidade, comparabilidade, quantificação e } \\
\text { objetividade }\end{array}$ \\
\hline 2 & $\begin{array}{l}\text { DeLone e } \\
\text { McLean, (2003) }\end{array}$ & E-commerce & $\begin{array}{l}\text { completude, compreensibilidade, personalização, } \\
\text { relevância e segurança }\end{array}$ \\
\hline 3 & $\begin{array}{l}\text { Wixon e Todd } \\
(2005)\end{array}$ & $\begin{array}{l}\text { Sistemas de } \\
\text { Informação (SI) }\end{array}$ & completeza, precisão, formato e atualidade \\
\hline 4 & $\begin{array}{l}\text { Mckinney; Yoon e } \\
\text { Zahedi (2002) }\end{array}$ & E-commerce & $\begin{array}{l}\text { relevância, compreensibilidade, confiabilidade, adequação, } \\
\text { escopo e utilidade }\end{array}$ \\
\hline 5 & Lewis (1995) & $\begin{array}{l}\text { Questionários de } \\
\text { usabilidade em SI }\end{array}$ & - \\
\hline 6 & $\begin{array}{l}\text { Kim; Ferrin e Rao } \\
(2008)\end{array}$ & E-commerce & exatidão, utilidade, atualidade, suficiência, satisfação \\
\hline 7 & $\begin{array}{l}\text { Francis et al. } \\
(2004)\end{array}$ & $\begin{array}{l}\text { Informações } \\
\text { contábeis }\end{array}$ & \\
\hline 8 & Rai (2002) & - & - \\
\hline 9 & $\begin{array}{l}\text { Lederer et al } \\
(2000)\end{array}$ & Sistemas web & $\begin{array}{l}\text { relevância, precisão, atualidade, compreensibilidade, } \\
\text { exatidão }\end{array}$ \\
\hline 10 & $\begin{array}{l}\text { Lambert; Leuz e } \\
\text { Verrecchia (2007) }\end{array}$ & $\begin{array}{l}\text { Informações } \\
\text { contábeis }\end{array}$ & - \\
\hline
\end{tabular}

Fonte: dados da pesquisa na WoS

Observa-se no Quadro 3 que os contextos de aplicação dos artigos mais citados, em sua maioria, estão relacionados a área da computação, uma vez que contempla sistemas de informação, sistemas web e sites de e-commerce. Outra questão a ser considerada, são as dimensões utilizadas para a avaliação da qualidade da informação nestes contextos, onde características como relevância, compreensibilidade, atualidade e precisão se repetem em quatro dos seis estudos mais citados.

Segundo Lee et al. (2002), quanto menos complexo e mais objetivo for o processo de avaliação, mais assertivo será o seu resultado. Percebe-se que esta premissa é seguida pelos outros modelos propostos, uma vez que o número de dimensões diminui consideravelmente nas mesmas. 


\section{Considerações finais}

Os resultados do estudo bibliométrico básico evidenciaram características elementares dos estudos sobre qualidade da informação, tais como as palavras-chave de maior ocorrência e a evolução da abordagem do tema na literatura.

Verificou-se que o número de publicações na Web of Science se encontra em uma curva crescente, totalizando 1.326 entre os anos de 1989 a 2016, o que demonstra um aumento de interesse e relevância do tema na literatura qualificada que foi analisada.

A partir da análise das palavras-chave, foi possível observar a interdisciplinaridade do tema, já que foram encontrados 2.536 termos distintos, relacionados às mais diversas áreas do conhecimento.

Considera-se que uma das maiores contribuições que puderam ser identificadas é a listagem com os 10 artigos mais citados na base, seus autores, os periódicos em que foram publicados, o ano e o número de citações de cada um deles. Também foram apresentados de forma sucinta, o objetivo dos mesmos e as dimensões da qualidade da informação contempladas nas diferentes publicações, possibilitando uma visão geral dos estudos mais citados. Tais dados podem fomentar desenvolvimentos futuros lastreados em dados consolidados de indexação numa base relevante e não podem ser desconsiderados quando se trata do tema da "qualidade da informação".

Após a análise dos artigos mais citados, percebeu-se que alguns deles não faziam referência direta a 'qualidade da informação', e sim tratavam do tema de forma secundária como é o caso das publicações de Lewis (1995), Francis et al. (2002) e Lambert; Leuz e Verrecchia (2007), porém continham o termo utilizado na busca em seus resumos e assim fizeram parte dos resultados. Acredita-se que da mesma forma que estas publicações, outras com essa característica foram contabilizadas no total de artigos encontrados, o que pode sugerir que o resultado não contemple exclusivamente estudos focados especificamente na área da qualidade da informação. Essa questão pode ser melhor explorada em estudos futuros.

Por este motivo, sugere-se como trabalhos futuros a aplicação do mesmo protocolo de busca na base de dados Web of Science, porém utilizando o campo "título", assim será possível uma comparação entre os resultados obtidos e será possível verificar se a hipótese de que a alteração do campo de busca, resultará em resultados direcionados especificamente ao tema qualidade da informação.

Outra possibilidade de trabalho futuro seria a realização de levantamento semelhante em outras bases de dados, como por exemplo a Scopus ou a Library and Information Science 
Abstracts (LISA), esta última focada em publicações direcionadas a área da Ciência da Informação.

Por fim, considera-se que os resultados evidenciaram dados relevantes para 0 desenvolvimento do tema nas diversas perspectivas dos stakeholders (usuários, desenvolvedores, avaliadores...), destacando-se particularmente a publicação de DeLone e McLean, (1992), a mais citada entre as 10 e também a primeira a ser publicada, que apresenta ao todo 23 dimensões da qualidade da informação a serem avaliadas e que podem ser reduzidas, agrupadas, integradas ou mesmo ser parâmetro de comparação com as outras propostas.

\section{Referências}

AROUCK, Osmar. Atributos de qualidade da informação. In: ENCONTRO NACIONAL DE PESQUISA EM CIÊNCIA DA INFORMAÇÃO, 12., 2011, Brasília. Anais eletrônicos... Brasília, 2011. Disponível em: http://enancib.ibict.br/index.php/enancib/enancibXII/paper/view/874. Acesso em: 25 maio 2017.

BATINI, Carlo et al. Methodologies for Data Quality Assessment and Improvement. ACM Computing Surveys, n. 3 , v. 41, p. 1-52. 2009. Disponível em: http://dl.acm.org/citation.cfm?id=1541883. Acesso em: 25 mai. 2017.

BIZER, Christian; CYGANIAK, Richard. Quality-driven information filtering using the WIQA policy framework. Web Semantics, v. 7, n. 1, p. 1-10, 2009Disponível em: http://www.sciencedirect.com/science/article/pii/S157082680800019X Acesso em: 3 jun. 2017.

CALAZANS, Angélica Toffano Seidel. Qualidade da informação: conceitos e aplicações. Transinformação, Campinas, v. 20, n. 1, p. 29-45,2008. Disponível em: http://www.scielo.br/pdf/tinf/v20n1/03.pdf Acesso em: 3 mar. 2017.

DELONE, William H.; MCLEAN, Ephraim R. Information systems success: The quest for the dependent variable. Information Systems Research, v. 3, n. 1, p. 60-95,1992. Disponível em: https://pdfs.semanticscholar.org/a041/45f1ca06c61f5985ab22a2346b788f343392.pdf Acesso em: 22 maio 2017.

DELONE, William H.; MCLEAN, Ephraim R. The DeLone and McLean Model of Information Systems Success: A Ten-Year Update. Journal of Management Information Systems, v. 19, n. 4, p. 9-30, 2003. Disponível em: http://dl.acm.org/citation.cfm?id=1289767 Acesso em: 3 jun. 2017.

FRANCIS, Jennifer et al. Costs of Equity and Attributes. The Accounting Review, v. 79, n. 4, 2015, $\quad$ p. 967-1010. Disponível em: $<$ http://econ.au.dk/fileadmin/Economics Business/Education/Summer University 2012/6 308 Advanced Financial Accounting/Advanced Financial Accounting/7/flos-jae-2004.pdf Acesso em: 22 jun. 2017. 
KIM, Dan J. et al. A trust-based consumer decision-making model in electronic commerce: The role of trust, perceived risk, and their antecedents. Decision Support Systems, v. 44, n. 2, p. 544-564,2008. Disponível em: http://www.sciencedirect.com/science/article/pii/S0167923607001005 Acesso em: 26 jun. 2017.

LAMBERT, Richard A.; LEUZ, Christian; VERRECCHIA, Robert E. Accounting information, disclosure, and the cost of capital. Journal of Accounting Research, v. 45, n. 2, p. 385-420, 2007. Disponível em: https://papers.ssrn.com/sol3/papers.cfm?abstract id=823504 Acesso em: 26 jun. 2017.

LEDERER, Albert L. et al. The technology acceptance model and the World Wide Web. Decision Support Systems, v. 29, n. 3, p. 269-282, 2000. Disponível em: https://pdfs.semanticscholar.org/21b4/f1e91ea6056be14761ce46be587d5ae2c7ad.pdf Acesso em: 20 jun. 2017.

LEE, Yang W. et al. AIMQ: A methodology for information quality assessment. Information and Management, v. 40, n. 2, p. 133-146, 2002. Disponível em: http://mitiq.mit.edu/Documents/Publications/TDQMpub/2002/AIMQ.pdf Acesso em: 20 maio 2017.

LEWIS, James R. IBM Computer Usability Satisfaction Questionnaires: Psychometric Evaluation and Instructions for Use. International Journal of Human-Computer Interaction, v. 7, n. 1, p. 57-78, $1995 . \quad$ Disponível em: https://www.researchgate.net/publication/200085994 Lewis JR IBM Computer Usability Sa tisfaction Questionnaires Psychometric Evaluation and Instructions for Use International Journal of Human-Computer Interaction 71 57-78 Acesso em: 22 jun. 2017.

LUCAS, Alexandre; VIERA, Angel Freddy Godoy; PINTO, Adilson Luiz. Análise da produção científica sobre inteligência de negócios. In: ENCONTRO NACIONAL DE PESQUISA EM CIÊNCIA DA INFORMAÇÃO, 16., 2013, João Pessoa. Anais eletrônicos... João Pessoa: 2013. Disponível em:

http://www.ufpb.br/evento/Iti/ocs/index.php/enancib2015/enancib2015/paper/viewFile/281 8/1085 Acesso em: 7 jun. 2017.

MCKINNEY, Vicki; YOON, Kanghyun; ZAHEDI, Fatemeh. The measurement of Web-customer satisfaction: An expectation and disconfirmation approach. Information Systems Research, v. 13, n. 3, p. 296-315, 2002. Disponível em: http://dl.acm.org/citation.cfm?id=769625 Acesso em: 18 abr. 2017.

NEHMY, Rosa Maria Quadros; PAIM, Isis. A desconstrução do conceito de "qualidade da informação". Ciência da Informação, Brasília, v. 27, n. 1, p. 36-45, 1998. Disponível em: http://www.scielo.br/pdf/ci/v27n1/05.pdf Acesso em: 28 mar. 2017.

PAIM, Isis et al. Problematização do conceito "Qualidade" da Informação. Perspectivas em Ciência da Informação, Belo Horizonte, v. 1, n. 1, p. 111-119, 1996. Disponível em http://portaldeperiodicos.eci.ufmg.br/index.php/pci/article/view/8/27 Acesso em: 30 mar. 2017.

PORTAL DE PERIÓDICOS DA CAPES/MEC. Disponível em: http://www.periodicos.capes.gov.br/?option=com pcollection \&mn=70\&smn=79\&cid=81 Acesso em: 7 jun. 2017. 
RIBEIRO, Claudio José Silva. Big Data: os novos desafios para o profissional da informação. Informação \& Tecnologia, João Pessoa, v. 1, n. 1, p. 96-105, 2014. Disponível em: http://periodicos.ufpb.br/index.php/itec/article/view/19380/11156 Acesso em: 19 abr. 2017.

VALENTE, Nelma T. Zubek; FUJINO, Asa. Atributos e dimensões de qualidade da informação nas Ciências Contábeis e na Ciência da Informação: um estudo comparativo. Perspectivas em Ciência da Informação, Belo Horizonte, v. 21, n. 2, p. 141-167, 2016. Disponível em: http://portaldeperiodicos.eci.ufmg.br/index.php/pci/article/view/2530/1761 Acesso em: 16 mar. 2017.

WANG, Richard Y.; STRONG, Diane M. Beyond Accuracy: What Data Quality Means to Data Consumers. Journal of Management Information System, v. 12, n. 4, p. 5-34, 1996. Disponível em: $\quad$ http://mitiq.mit.edu/Documents/Publications/TDQMpub/14 Beyond Accuracy.pdf Acesso em: 16 abr. 2017.

WIXOM, Barbara H.; TODD, Peter A. A theoretical integration of user satisfaction and technology acceptance. Information Systems Research, v. 16, n. 1, p. 85-102, 2005. Disponível em: $\quad$ https://pdfs.semanticscholar.org/95ea/e3a82d9d1b01ca4eafd4922cd0f719517c32.pdf Acesso em: 7 jun. 2017 